\title{
Ultra-Thin, Strong and Cell-Adhesive Agarose-Based Membranes Engineered as Substrates for Corneal Endothelial Cells
}

Wei Yang Seow, ${ }^{1, *}$ Karthikeyan Kandasamy, ${ }^{1}$ Gary S. L. Peh, ${ }^{2,3}$ Jodhbir S. Mehta ${ }^{2,3,4}$ and William Sun ${ }^{1, *}$

${ }^{1}$ Institute of Bioengineering and Nanotechnology, 31 Biopolis Way, \#07-01, Singapore 138669.

${ }^{2}$ Tissue Engineering and Stem Cell Group, Singapore Eye Research Institute, Singapore.

${ }^{3}$ Duke-NUS Graduate Medical School, Singapore.

${ }^{4}$ Singapore National Eye Centre, Singapore.

*Corresponding author. E-mail: wyseow@ibn.a-star.edu.sg :wsun@ibn.a-star.edu.sg

This Supporting Information (SI) consists of 9 pages, 5 Figures and 1 video. 


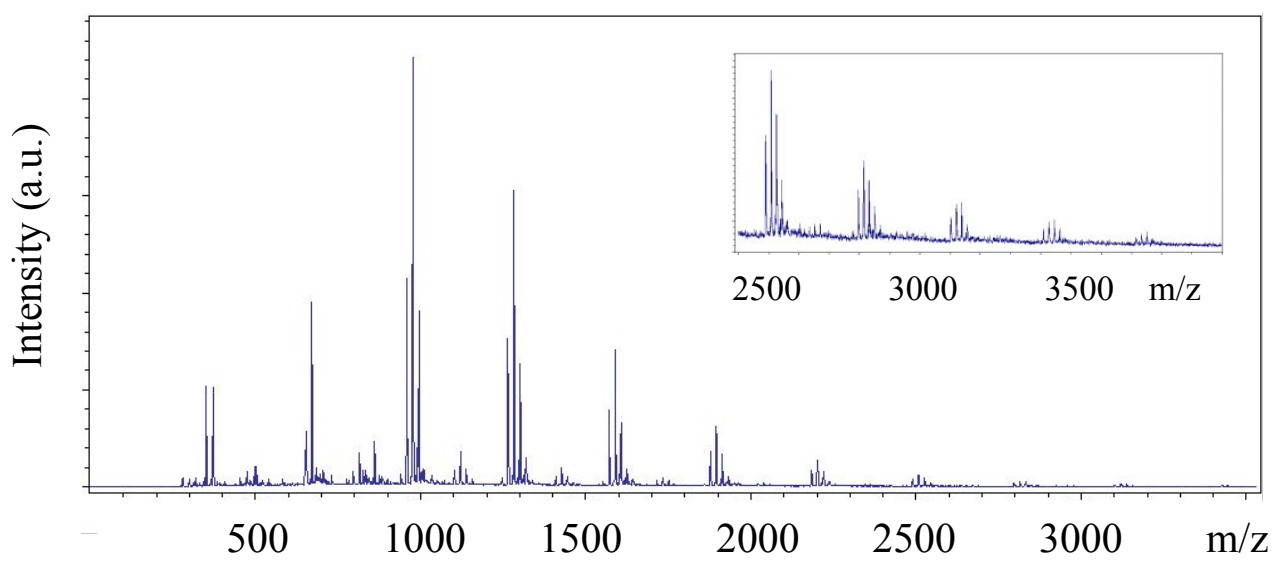

Figure S1. The stability of agarose was tested under acidic conditions similar to those typically used for the removal of pbf and OtBu peptide protection groups. Agarose powder was stirred in $95 \%$ TFA at $25^{\circ} \mathrm{C}$ for 2 hours. Agarose was then precipitated in ethanol, washed and isolated by filtration. After vacuum drying overnight, the product was dissolved in water $(10 \mathrm{mg} / \mathrm{mL})$ but did not form a gel upon cooling. This provided early evidence of its reduced molecular weight due to degradation by acid hydrolysis. $\alpha$-Cyano-4hydroxycinnamic acid (Sigma Aldrich, Singapore) was mixed in as the matrix and $2 \mu \mathrm{L}$ of sample was deposited and air-dried on a steel target plate using the dried-droplet method. Matrix-assisted laser desorption/ionization time-of-flight (MALDI-TOF, Bruker ultrafleXtreme, MA, USA) mass spectrometry was used to quantify the molecular weight distribution of agarose. The agarose polysaccharide was degraded into oligosaccharides and the disaccharide repeat unit of $\sim 306$ Da was clearly detected. The inset shows the magnified spectrum at higher $\mathrm{m} / \mathrm{z}$. We note that MALDI-TOF gave good signals with degraded agarose oligosaccharide, but not with intact agarose polysaccharide. For reference, the molecular weight of native agarose was estimated to be $M_{n}=181 \mathrm{kDa}$ and $M_{w}=284 \mathrm{kDa}$ by GPC analysis, relative to polystyrene standards and with DMF as the solvent. 

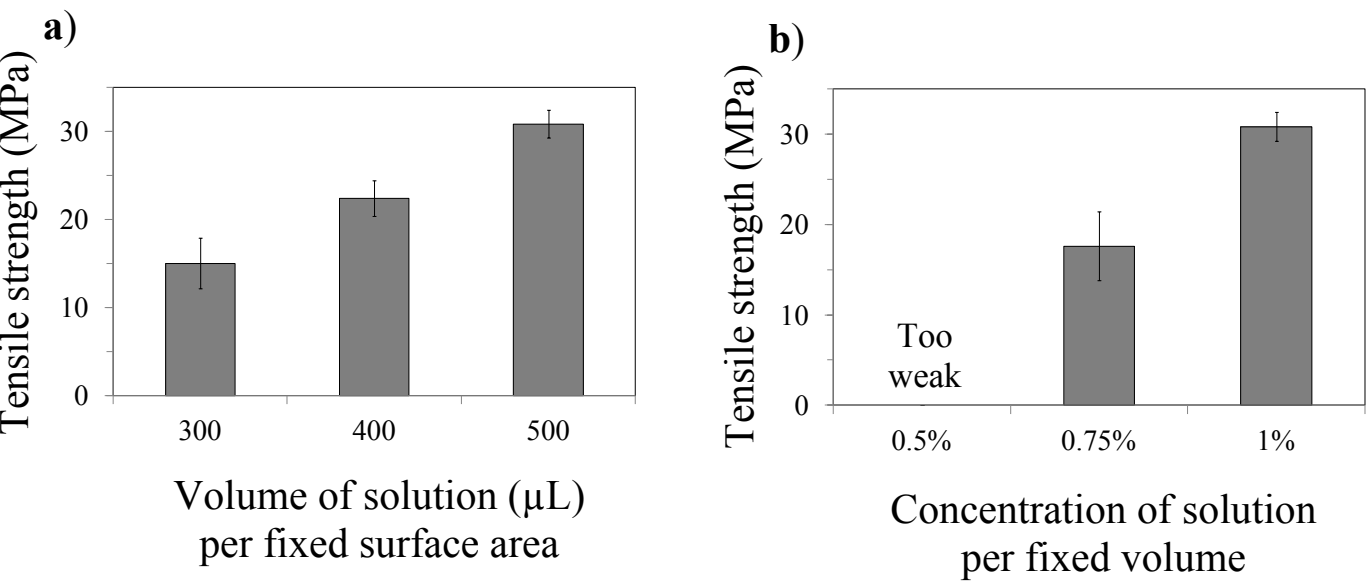

Figure S2. Optimization of conditions used to prepare the ultra-thin membrane. a) Effect of the volume of solution : surface area of chamber on the tensile strength of membranes. AK2 was first dissolved in water $(10 \mathrm{mg} / \mathrm{mL}$, or $1 \%)$ at $100^{\circ} \mathrm{C} .300,400$ or $500 \mu \mathrm{L}$ was then dispensed into 4-well chambered slides with removable chamber tops (SPL Life Sciences, Korea). Therefore, only volume was varied, while concentration and surface area were fixed. Membranes were formed as described previously and subjected to tensile testing. As expected, tensile strength increased with volume of solution used and $500 \mu \mathrm{L}$ was selected for future experiments to obtain membranes with greater reproducibility and ease-ofhandling. b) Effect of concentration on the tensile strength of membranes. AK2 was dissolved at different concentrations $(0.5 \%, 0.75 \%$ and $1 \%)$ and $500 \mu \mathrm{L}$ was dispensed into the 4 -well chamber slides. Here, concentration was varied, while volume and surface area were maintained. Tensile testing revealed that membranes formed with $0.5 \%$ solution were too weak to be even tested. Membranes formed with $0.75 \%$ solution were strong enough to be tested but required careful handling. Eventually, conditions of $500 \mu \mathrm{L}$ of a $1 \%$ solution were determined to be optimal for a 4 -well chamber slide for future experiments. Conditions were scaled accordingly if a larger sheet of membrane was required. 


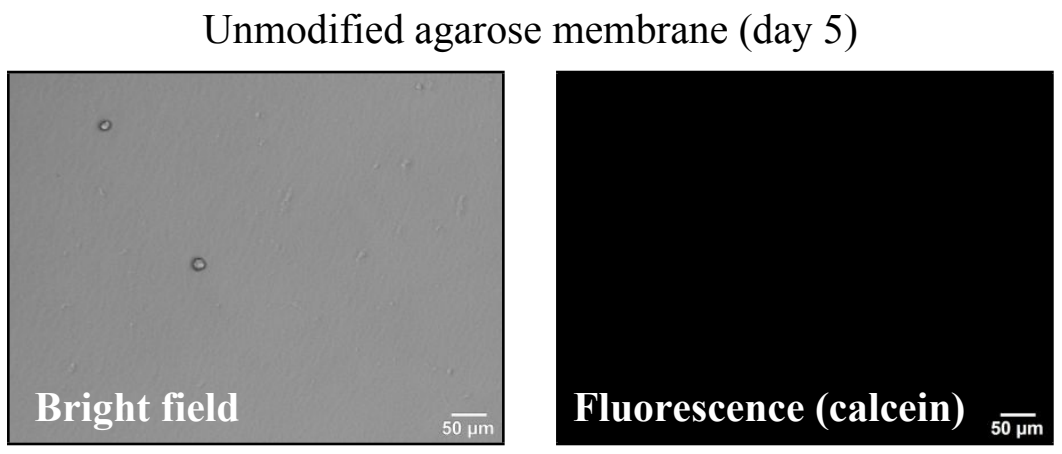

Figure S3. RCEC was seeded onto membranes formed using unmodified agarose and imaged after 5 days. Both the bright field and fluorescence images showed the absence of adherent cells. The cells were most probably washed away during media change prior to imaging. 


\section{Physical blend}

$5.7 \mathrm{wt} \%$ gelatine

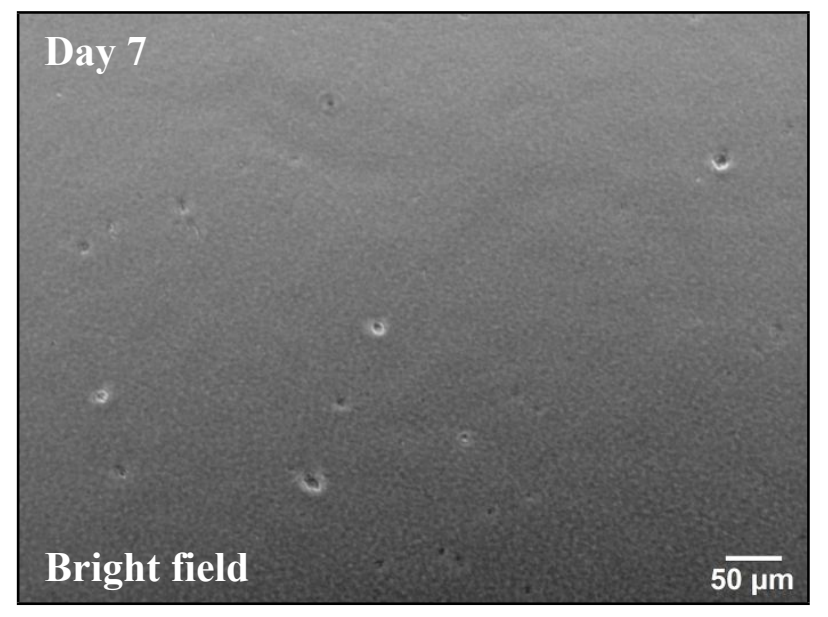

Figure S4. Gelatine $(5.7 \mathrm{wt} \%)$ was simply mixed in (physical blend, no chemical conjugation) with agarose to form membranes as described previously. RCEC were then seeded onto the membrane, as before, and media was changed every 2-3 days. Cells were not observed to be adherent on day 7 . 


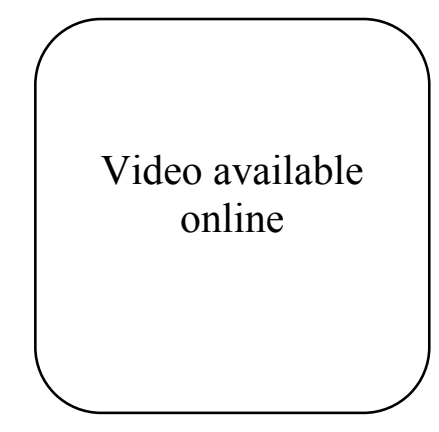

Figure S5. The membranes possessed the flexibility required for successful application. In this video, AG1 membrane was folded in half, inserted into a cadaveric rabbit anterior chamber and then unfolded in a mock-up procedure reminiscent of an endothelial keratoplasty. Note that the membrane has been artificially stained blue to improve contrast and visibility. 


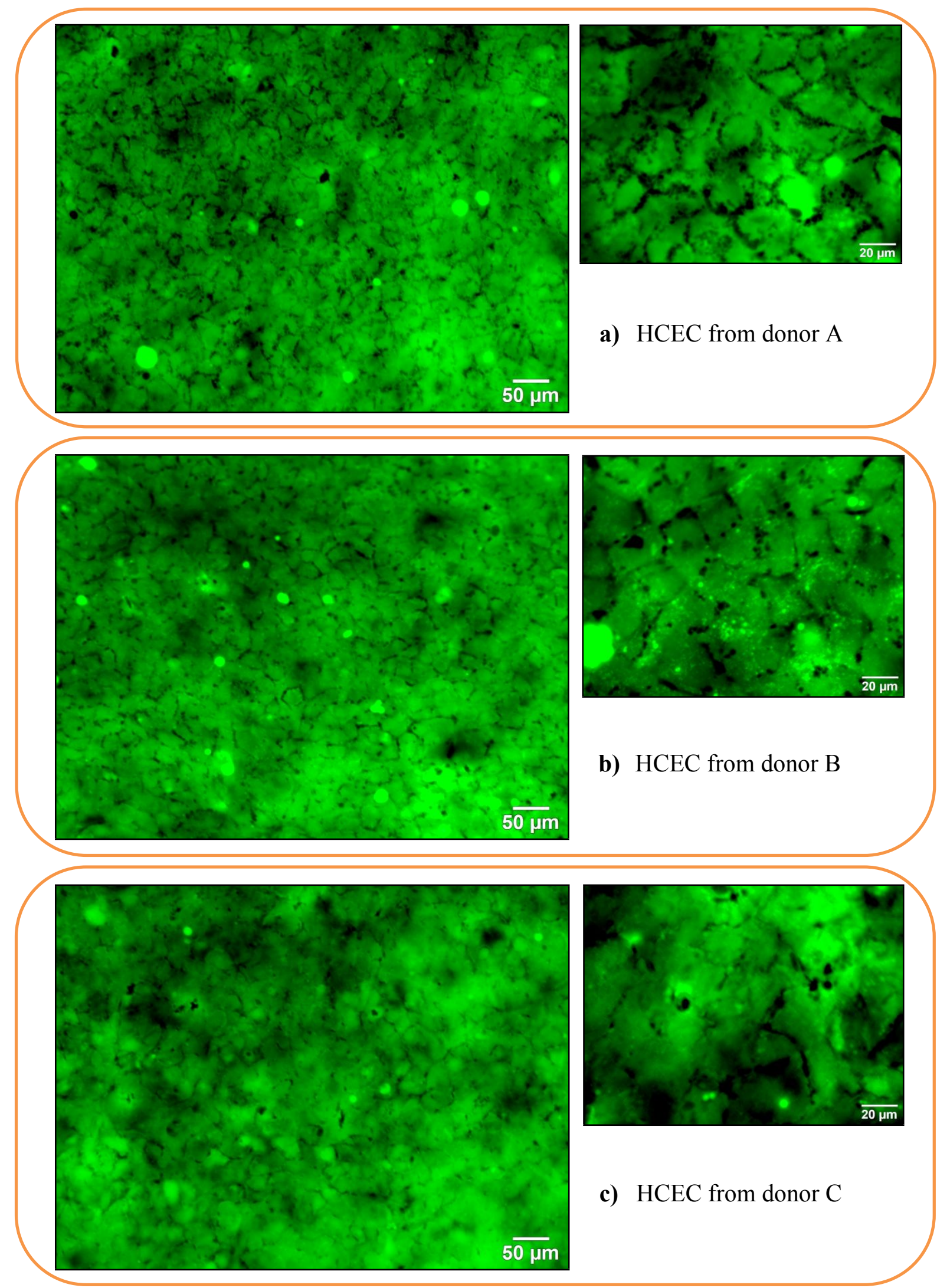

Figure S6. a-c) Human corneal endothelial cells (HCEC) from three separate donors successfully attached to AG1 membranes and remained viable, as evidenced by the positive calcein staining. 
Ham's F12, Medium 199, Human Endothelial-SFM, TrypLETM Select (TS), gentamicin, amphotericin B, penicillin and streptomycin were purchased from Life Technologies (California, USA). Collagen IV from human placenta was from Sigma (Missouri, USA). Human recombinant basic fibroblast growth factor (HrFGF), and Rhoassociated, coiled-coil protein kinase inhibitor (ROCKi), Y-27632, were from Miltenyi Biotec (Bergisch Gladbach, Germany). Liberase TH was from Roche (Mannhein, Germany). EquaFetal ${ }^{\circledR}$ was from Atlas Biologicals (Colorado, USA). Insulin/Transferrin/Selenium (ITS) was from Corning (NY, USA), and ascorbic acid from Avantor (Pennsylvania, USA).

The use of cadaver corneal tissues was approved by Singhealth centralized institutional review board (Ref: 2016/2839). Research-grade human cadaver corneal tissues (three donors aged 18, 8 and 13, all with HCEC density $\geq 2,000$ cells $/ \mathrm{mm}^{2}$ ) were procured from Lions Eye Institute for Transplant and Research (Florida, USA) with informed consent from the next of kin. All research performed with HCEC was in accordance with the principles outlined in the Declaration of Helsinki. Corneo-scleral donor tissues were maintained in Optisol-GS (Bausch \& Lomb, New York, USA) at $4^{\circ} \mathrm{C}$ until use.

HCEC was maintained in a humidified atmosphere at $37^{\circ} \mathrm{C}$ and $5 \% \mathrm{CO}_{2}$. The isolation and culture of HCEC was achieved using a dual media culture approach, as described previously. ${ }^{1,2}$ Briefly, HCEC was isolated using a two-step enzymatic treatment. Isolated cellular clusters of HCEC were rinsed twice before being seeded onto collagencoated culture vessels and established in a corneal endothelial maintenance/stabilization medium (M5-Endo; Human Endothelial-SFM supplemented with 5\% serum) overnight. Subsequently, HCEC was cultured in a proliferative medium (M4-F99; Ham's F12/M199, 5\% serum, $20 \mu \mathrm{g} / \mathrm{ml}$ ascorbic acid, 1x ITS, and $10 \mathrm{ng} / \mathrm{ml} \mathrm{HrFGF).} \mathrm{Upon} \mathrm{reaching} \mathrm{80-90 \%}$ confluency, cells were re-exposed to M5-Endo for at least two days, before being subcultured via single-cell dissociation using TS. Dissociated HCEC was seeded at $\geq 1.0 \times 10^{4}$ 
cells $/ \mathrm{cm}^{2}$ on collagen-coated surfaces and expanded to the second passage. The cells were then dissociated into single-cells and seeded at 2,000 cells $/ \mathrm{mm}^{2}$ onto the agarose membrane. HCEC was maintained in M5-Endo media for seven days, before labelling with the CalceinAM dye and imaged with an inverted fluorescence microscope (IX-83, Olympus, Japan).

\section{References}

(1) Peh, G.S.L.; Chng, Z.; Ang, H.-P.; Cheng, T.Y.D.; Adnan, K.; Seah, X.-Y.; George, B.L.; Toh, K.-P.; Tan, D.T.; Yam, G.H.F.; Colman, A.; Mehta, J.S. Propagation of human corneal endothelial cells: a novel dual media approach. Cell Transplantation 2015, 24, 287-304, DOI: 10.3727/096368913X675719.

(2) Peh, G.S.L.; Ang, H.-P.; Lwin, C.N.; Adnan, K.; George, B.L.; Seah, X.-Y.; Lin, S.J.; Bhogal, M.; Liu, Y.-C.; Tan, D.T.; Mehta, J.S. Regulatory compliant tissueengineered human corneal endothelial grafts restore corneal function of rabbits with bullous keratopathy. Scientific Reports 2017, 7: 14149, DOI: 10.1038/s41598-01714723-z. 